\title{
Pancreatic Precancerous Condition
}

National Cancer Institute

\section{Source}

National Cancer Institute. Pancreatic Precancerous Condition. NCI Thesaurus. Code C7412.

A pathologic process that arises from the pancreas and has the potential to evolve into a malignant neoplasm. 